\title{
Quantum Beams for New Aspects in Magnetic Materials and Magnetism
}

\author{
Robert Georgii ${ }^{1, *(D)}$ and Klaus-Dieter Liss ${ }^{2}$ (D) \\ 1 Heinz Maier-Leibnitz Zentrum (MLZ), Technische Universität München, Lichtenbergstr. 1, \\ 85747 Garching, Germany \\ 2 Guangdong Technion-Israel Institute of Technology, 241 Daxue Lu, Jinping Qu, Shantou 515063, \\ Guangdong, China; kdl@gtiit.edu.cn \\ * Correspondence: robert.georgii@frm2.tum.de; Tel.: +49-89-289-14986
}

Received: 7 October 2019; Accepted: 28 October 2019; Published: 25 November 2019

Welcome to the special issue of Quantum Beam Science on "Magnetic Materials and Magnetism". Despite a long tradition of research in magnetism, exciting new developments are still regularly being reported. In this issue, only a very small selection of these developments over recent years will be presented. Particularly, new progress in probes and sources is occurring, which promises a better understanding of magnetic novel states and systems. This proves again that the discovery of new concepts goes in parallel with combining different methods or the use of new methods. This is reviewed in [1], showing how muons with high intensity from a new muon source at the Chinese spallation source will bring new insights in the field of magnetic materials and magnetism. By combining atom probe tomography (APT) with quasielastic neutron scattering using $\mathrm{N}(\mathrm{R}) \mathrm{SE}$, the evolution of the spin glass phase in the presence of ferro- and antiferromagnetic clusters in the cluster spin glass Fe17.8Cr82.2 has been characterized in [2]. The authors interpreted the decay of spin fluctuations in parallel processes to be finite and considered that cluster-type spin glasses may generally be classified based on the value of the parameter $k$, which is a measure for the number of parallel processes at low $\mathrm{T}$.

Another field where a new set-up for measuring a well-known system generated a completely new view on magnets with non-centrosymmetric structure are magnetic skrymions. The understanding and research on these topological spin vortices developed dramatically since their first theoretical foundations in the 1990s and their discovery in the non-centrosymmetric MnSi in the first decade of this century. The number of publications reached more than 2000 and citations rose to some thousands per year recently. The early developments in this field are the topic of the review [3]. Interestingly, Muon spin rotation $(\mu \mathrm{SR})$ spectra recorded for manganese silicide $\mathrm{MnSi}$ in [4] showed that the magnetic structures of $\mathrm{MnSi}$ in zero-field at a low temperature and in the conical phase near the magnetic phase transition deviate from the expected helical and conical structures. This highlights the importance of comparing the results using different probes for obtaining the full picture of magnetic phenomena. Thus, using an actual synchrotron technique, the lensless coherent small-angle scattering with circularly polarized soft $X$-rays offers the possibility to obtain a real space picture of the magnetic texture of FeGe as reported in [5]. Here, real space images of the helical and skyrmionic texture were reproduced adding the missing phase information of the scattering techniques to the overall picture.

Other interesting magnetic systems are the triangular frustrated magnets. In [6], neutron scattering was used to determine the critical exponent $\beta$ describing the universal behavior of magnetization near the Curie temperature. The measurements were performed in $\mathrm{YMnO}_{3}$ in undoped and doped material both under ambient and high pressure. The low value of $\beta \approx 0.20$ found consistently in all these measurements is in agreement with that of the similar material $\mathrm{VCl}_{2}$. A possible explanation for this is that frustrated triangular magnets are in a separate universality class with an unusually low value of $\beta$.

Conflicts of Interest: The authors declare no conflict of interest. 


\section{References}

1. Tang, J.; Ni, X.; Ma, X.; Luo, H.; Bao, Y.; Yuan, Y.; Chen, Y.; Chen, Y.; Deng, F.; Dong, J.; et al. EMuS Muon Facility and Its Application in the Study of Magnetism. Quantum Beam Sci. 2018, 2, 23. [CrossRef]

2. Wagner, J.N.; Häußler, W.; Holderer, O.; Bauer, A.; Shapiro, S.M.; Böni, P. Influence of Concentration Fluctuations on Relaxation Processes in Spin Glasses. Quantum Beam Sci. 2018, 2, 26. [CrossRef]

3. Georgii, R.; Weber, T. The Helical Magnet MnSi: Skyrmions and Magnons. Quantum Beam Sci. $2019,3,4$. [CrossRef]

4. Dalmas de Réotier, P.; Yaouanc, A.; Amato, A.; Maisuradze, A.; Andreica, D.; Roessli, B.; Goko, T.; Scheuermann, R.; Lapertot, G. On the Robustness of the MnSi Magnetic Structure Determined by Muon Spin Rotation. Quantum Beam Sci. 2018, 2, 19. [CrossRef]

5. Ukleev, V.; Yamasaki, Y.; Morikawa, D.; Kanazawa, N.; Okamura, Y.; Nakao, H.; Tokura, Y.; Arima, T.H. Coherent Resonant Soft X-ray Scattering Study of Magnetic Textures in FeGe. Quantum Beam Sci. 2018, 2, 3. [CrossRef]

6. Holm-Dahlin, S.; Janas, S.; Kreisel, A.; Pomjakushina, E.; White, J.S.; Fennell, A.L.; Lefmann, K. The Magnetic Phase Transition and Universality Class of $\mathrm{h}-\mathrm{YMnO}_{3}$ and $\mathrm{h}-\left(\mathrm{Y}_{0.98} \mathrm{Eu}_{0.02}\right) \mathrm{MnO}_{3}$ Under Zero and Applied Pressure. Quantum Beam Sci. 2018, 2, 16. [CrossRef]

(C) 2019 by the authors. Licensee MDPI, Basel, Switzerland. This article is an open access article distributed under the terms and conditions of the Creative Commons Attribution (CC BY) license (http://creativecommons.org/licenses/by/4.0/). 